\title{
De novo assembly of the desert tree Haloxylon ammodendron (C. A. Mey.) based on RNA-Seq data provides insight into drought response, gene discovery and marker identification
}

Yan Long ${ }^{1 \dagger}$, Jingwen Zhang ${ }^{1 \dagger}$, Xinjie Tian ${ }^{1}$, Shanshan Wu', Qiong Zhang ${ }^{2}$, Jianping Zhang ${ }^{2}$, Zhanhai Dang ${ }^{2^{*}}$ and Xin Wu Pei ${ }^{1 *}$

\begin{abstract}
Background: Haloxylon ammodendron (C. A. Mey.) is widely distributed across a range of habitats, including gravel desert, clay desert, fixed and semi-fixed sand, and saline land in Asian and African deserts. To date, no genomic information or expressed sequence tag-simple sequence repeat (EST-SSR) marker has been reported for $\mathrm{H}$. ammodendron plants.

Results: Using Illumina sequencing technology, we generated over two billion bases of high-quality sequence data on H. ammodendron and conducted de novo assembly and annotation of genes without prior genome information. These reads were assembled into 79,918 unigenes (mean length $=728$ bp). Based on similarity searches comparing these unigenes with known proteins in the non-redundant (nr) protein database, 25,619 unigenes were functionally annotated with a cut-off E-value of $10^{-5}$. In addition, DGE reads were mapped to the assembled transcriptome for gene expression analysis under drought stress. In total, 1,060 differentially expressed genes were identified. Among these genes, 356 genes were upregulated after drought treatment, and 704 genes were downregulated. We used the KEGG database to annotate these drought-induced genes; 207 unigenes were identified in the KEGG pathway annotation, and approximately $12.1 \%$ of the unigenes with known function fell into categories related to fatty acid metabolism, starch and sucrose metabolism, and nitrogen metabolism, suggesting that these pathways or processes may be involved in the drought response. Together, a total of 35 drought-inducible transcription factors were identified, including WRKY, MYB and bZIP family members.

Conclusions: Our study is the first to provide a transcriptome sequence resource for $\mathrm{H}$. ammodendron plants and to determine its digital gene expression profile under drought conditions using the assembled transcriptome data for reference. These data provide a valuable resource for genetic and genomic studies of desert plants under abiotic conditions.
\end{abstract}

Keywords: Haloxylon ammodendron, Drought, Transcriptome, Digital gene expression, EST-SSR

\footnotetext{
*Correspondence: 13669338239@163.com; peixinwu@caas.cn

${ }^{\dagger}$ Equal contributors

${ }^{2}$ Crop Institute, Gansu Agricultural Academy, Lanzhou 730070, China

${ }^{1}$ Institute of Biotechnology, Chinese Academy of Agricultural Sciences,

Beijing 100081, China
}

\section{Biomed Central}

(c) 2014 Long et al.; licensee BioMed Central. This is an Open Access article distributed under the terms of the Creative Commons Attribution License (http://creativecommons.org/licenses/by/4.0), which permits unrestricted use, distribution, and reproduction in any medium, provided the original work is properly credited. The Creative Commons Public Domain Dedication waiver (http://creativecommons.org/publicdomain/zero/1.0/) applies to the data made available in this article, unless otherwise stated. 


\section{Background}

Drought is one of the most common environmental abiotic stresses in the world. To adapt to environmental changes, plants have a variety of physiological responses and defence systems for withstanding drought conditions. The regulatory mechanism in higher plants has been analysed by studying a number of genes responding to drought stress at the transcriptional level [1,2]. In Arabidopsis thaliana, for example, thousands of genes are thought to be involved in abiotic stress [3]. In general, drought stress-inducible genes have been classified into two groups: one group that directly protects plants against environmental stresses, and a second group that regulates gene expression networks and signalling in stress responses, such as in response to drought or salt stress [2]. Recently, progress has been made in analysing the functions of stress-inducible genes, not only to understand the mechanisms of drought stress but also to improve the drought tolerance of plants by gene transfer. Genetic studies have identified many transcription factors that are extensively involved in the regulatory network of drought-inducible genes [2,3], including, for example, the NAC [4], WRKY [5], and MYB families [6].

As a xerophytic desert tree, Haloxylon ammodendron has great drought and salt resistance; thus, it plays an important role in the maintenance of the structure and function of the entire ecosystem in which it grows. $H$. ammodendron reduces wind speed and ameliorates the forest microclimate, thereby facilitating the settlement and growth of other desert plants [7]. H. ammodendron is widely distributed in a variety of habitats, including gravel desert, clay desert, fixed and semi-fixed sand, and saline land in the Asian and African deserts [8]. In China, about $56 \%$ of $H$. ammodendron is found in Xinjiang province, $40 \%$ in Inner Mongolia province, and the remaining 4\% in Qinghai and Gansu provinces [9]. At present, most research examining the drought tolerance of $H$. ammodendron focuses on physiology [10-12] and on the identification of specific genes. Some important drought-related genes have been cloned using RT-PCR and the RACE method, including the CMO [13], ARF1 [14] and EF-hand CaBP [15] genes. However, to our knowledge, no study involving large-scale drought-related gene screening and EST-SSR identification has been published to date.

Genome-wide analyses have dramatically improved the efficiency of gene discovery. With the advent of nextgeneration sequencing, large-scale transcriptome data has become available in both model and non-model species. Since Hegedus et al. [16] first used Solexa/Illumina's Digtal Gene Expression (DGE) system to study the zebrafish transcriptome after Mycobacterium marinum infection, RNA-Seq and DGE technology have been widely used to identify plant genes, including those expressed in stress condition $[17,18]$, related to important agronomic traits.
For example, in A. thaliana, about $30 \%$ of the transcriptome was found to be regulated by abiotic stress, and 2,409 genes were identified as being of great importance in cold, salt, and drought tolerance [3]. In Chinese cabbage, Yu et al. (2012) conducted transcriptome profiling by tag sequencing and by quantifying the expression of more than 10,000 genes in response to dehydration stress; these authors found that 28 genes in 37 transcription factors were involved in signal transduction and that the expression of 61 water- and osmosensing-responsive genes was significantly altered in response to water deficit [19].

Molecular markers play important roles in many activities involved in plant breeding, such as studies of genetic diversity [20], marker-assisted selections [21], and the identification of genes responsible for desirable traits [22]. Molecular markers have been widely used to map important genes and to assist with the breeding of trees. Many ESTSSR markers were developed using collected ESTs or highthroughput sequencing data. For example, in the rubber tree, Li et al. [23] used Hiseq2000 sequencing to sequence RNA from the bark of healthy rubber trees and got more than 30 million sequencing reads; after these sequences were assembled, 22,756 unigenes were obtained. A total of 39,257 EST-SSRs were then identified from these 22,756 unigenes. Finally, the PCR success ratio for the 110 randomly selected primers used in this study was 96.36\% [23]. In the date palm, Zhao et al. (2012) identified 4,609 ESTSSRs from 28,889 EST sequences, and after examining their random primers, these authors found that one third of their primers had polymorphisms in 12 different date palm cultivars [24].

In this study, the transcriptome from different tissues of drought-treated and control $H$. ammodendron plants were sequenced with Illumina paired-end sequencing technology. The resulting sequence data were assembled and annotated, DGE profiling was performed, and EST-SSR markers were developed. To our knowledge, this is the first systematic report on the transcriptome of $H$. ammodendron. This research is essential to understand the transcriptional changes underlying the drought response in $H$. ammodendron. The transcriptome data generated from our study provide a resource for gene annotation and discovery, the development of molecular markers, genomic and transcriptomic assembly, and the development of microarrays for $H$. ammodendron. In addition, the EST-SSR markers predicted and developed in this study enlarge the number of available molecular markers and may facilitate gene mapping, linkage map development, genetic diversity analysis, and markerassisted selection breeding in $H$. ammodendron.

\section{Results}

Illumina paired-end sequencing and de novo assembly To investigate the transcriptome of $H$. ammodendron under drought conditions, RNA was extracted from 
different tissues and sequenced using Illumina paired-end sequencing technology. In this study, a total of 74,011,190 raw sequencing reads with a length of $100 \mathrm{bp}$ each were generated from a 200 bp insert library. After removing adaptors and low quality data, 70,947,290 clean reads were obtained. The high-quality reads were then used to assemble the transcriptome data using Trinity software. Using overlapping information in high-quality reads, a total of 153,589 transcripts were generated, with an average length of 1,060 bp and an N50 of 1,812 bp. After compared the different transcripts representing one unigene, the longest length transcript for each unigene was extracted. So then a total of 79,918 unigenes were obtained. The average length was $728 \mathrm{bp}$, and transcripts with lengths of more than 500 bp accounted for about $37.15 \%$ of all transcripts (Table 1, Additional file 1).

\section{Annotation of all non-redundant unigenes}

For the validation and annotation of the assembled unigenes, all assembled unigenes were screened against the NCBI non-redundant (nr) and SWISS-PROT protein databases using the BLAST 2.2.28+ program with an Evalue threshold of 1e-5. Among 79,918 unigenes, 25,619 (32.05\%) were found to have significant similarity to 20,988 unique proteins. Of all the unigenes, 18,272 (22.86\%) with significant identities to SWISS-PROT proteins were matched with 11,269 unique protein accessions (Table 2). It was found that a smaller percentage was obtained when searching against the SWISS-PROT protein database rather than against the nr database. In total, BLAST searches identified 18,221 unique protein accessions from the $\mathrm{nr}$ and SWISS-PROT protein databases, suggesting that our Illumina paired-end sequencing had likely captured a substantial proportion of the drought-response genes in $H$. ammodendron.

\section{Functional classification by GO and COG}

In order to classify the functions of the predicted $H$. ammodendron unigenes, Gene ontology (GO), which is an internationally standardised gene functional classification system, was used. In total, 22,586 unigenes with BLAST matches to known proteins were assigned to GO classes using 1,734 functional terms (Table 2, Additional file 2). As shown in Figure 1A, the majority of the unigenes were assigned to the categories of biological processes $(58,097,45.69 \%)$, followed by cellular components $(6,085,29.81 \%)$ and molecular functions $(40,494,24.5 \%)$.
Under the category of biological processes, cellular processes $(13,279,22.86 \%)$ and metabolic processes $(12,707$, $21.87 \%$ ) were prominently represented, indicating that important cellular processes and metabolic activities occurred in $H$. ammodendron in response to drought. Under the classification of molecular functions, binding (13,141, $46 \%)$ and catalytic activities $(11,013,38.5 \%)$ were separately the first and second largest categories, respectively, whereas other categories, such as those for transporter activities, enzyme regulator activities, molecular transducer activities, and others, together contained only 4,410 unigenes representing $15.44 \%$ of the total number of unigenes. As for the cellular component, two categories, pertaining to cells and cell parts, accounted for approximately $39.19 \%$ of the cellular components that were identified; the organelle category accounted for approximately $14.14 \%$ of the cellular component unigenes, and the membrane and membrane part categories accounted for $21.13 \%$.

In order to predict and classify possible functions, all unigenes were aligned to the Cluster of Orthologous Groups (COG) database in which orthologous gene products were classified. Out of 25,619 unigenes with significant similarity to $\mathrm{nr}$ proteins in this study, 10,806 sequences were assigned to COG classifications (Figure 1B). Among the 25 COG categories, the cluster related to general function prediction $(1,877,17.37 \%)$ was the largest group, followed by those for posttranslational modification $(1,298,12.01 \%)$; translation, ribosomal structure and biogenesis $(806,7.46 \%)$; and signal transduction mechanisms (713, 6.6\%).

\section{Functional classifications using KEGG pathways}

The Kyoto Encyclopaedia of Genes and Genomes (KEGG) pathway database is a knowledge base for the systematic analysis of gene functions in terms of networks of genes and molecules in cells and their variants specific to particular organisms. To further analyse the transcriptome of $H$. ammodendron, all the unigenes were analysed with respect to the KEGG pathway database. Out of the 79,918 identified unigenes, 11,155 (13.96\%) with significant matches in the database were assigned to 5 main categories that included 265 KEGG pathways (Figure 2, Additional file 3$)$. Among the 5 main categories that were identified, metabolism was the category with the greatest number of unigenes $(5,326,47.75 \%)$, followed by genetic information $(2,044,18.39 \%)$, organismal systems $(1,781,16.02 \%)$,

Table 1 Summary of the Haloxylon ammodendron transcriptome

\begin{tabular}{|c|c|c|c|c|c|c|c|c|}
\hline \multirow[t]{2}{*}{ Category } & \multicolumn{4}{|l|}{ Number } & \multirow{2}{*}{$\begin{array}{l}\text { Total } \\
\text { number }\end{array}$} & \multirow{2}{*}{$\begin{array}{l}\text { Mean } \\
\text { length } \\
\text { (bp) }\end{array}$} & \multirow{2}{*}{$\begin{array}{l}\text { N50 } \\
\text { (bp) }\end{array}$} & \multirow{2}{*}{$\begin{array}{l}\text { Total } \\
\text { nucleotides }\end{array}$} \\
\hline & $200-500 \mathrm{bp}$ & 500-1 kbp & $1 \mathrm{k}-2 \mathrm{kbp}$ & $>2 \mathrm{kbp}$ & & & & \\
\hline Transcripts & 65,913 & 29,962 & 33,638 & 24,076 & 153,589 & 1,060 & 1,812 & $162,880,991$ \\
\hline Unigenes & 50,226 & 13,322 & 9,722 & 6,648 & 79,918 & 728 & 1,345 & $58,206,305$ \\
\hline
\end{tabular}


Table 2 Summary of the functional annotation of assembled unigenes

\begin{tabular}{lcc}
\hline Public database & $\begin{array}{c}\text { Number of } \\
\text { unigenes }\end{array}$ & Percentage (\%) \\
\hline Annotated in nr & 25,619 & 32.05 \\
Annotated in nt & 11,402 & 14.26 \\
Annotated in KO & 7,246 & 9.06 \\
Annotated in SWISS-PROT & 18,272 & 22.86 \\
Annotated in PFAM & 20,469 & 25.61 \\
Annotated in GO & 22,586 & 28.26 \\
Annotated in KOG & 9,680 & 12.11 \\
Annotated in all databases & 3,338 & 4.17 \\
Annotated in at least one database & 29,989 & 37.52 \\
Total Unigenes & 79,918 & 100 \\
\hline
\end{tabular}

cellular processes $(1022,9.19 \%)$ and environmental information processing $(982,8.83 \%)$. These results indicate that active metabolic processes were occurring in the drought treatment condition. As shown in Additional file 3, the KEGG metabolism category contained 12 sub-categories, including carbohydrate metabolism, nucleotide metabolism, biosynthesis involved in secondary metabolism, amino acid metabolism, lipid metabolism, and energy metabolism, among others.

\section{Analysis of differential gene expression during the drought process}

To reveal the molecular events occurring during the drought process, two digital gene expression (DGE) libraries were constructed using RNA from the two pools of control and drought treatment RNA samples and sequenced using Illumina technology. After Illumina sequencing and the removal of adaptors and bad-quality reads, approximately 11,019,037 and 12,958,245 reads were obtained for the two control replicates, and $13,915,918$ and $12,960,189$ reads were obtain for the two replicates for the drought-treated plants. We then mapped the clean reads to the transcriptome reference data, and a total of 57,695 and 55,998 unigene sequences were identified for the control replicates, and 58,788, and 57,596 unigene sequences were identified for the drought replicates. After calculating the expression level of each mapped unigene, a total of 1,060 unigenes were detected that had levels of expression that were significantly different between the drought-treated and control libraries. No homologue was found in the NCBI database for 469 differentially expressed unigenes. Both upregulation and downregulation of unigene expression occurred among the differentially expressed unigenes. Among all differentially expressed unigenes, 356 were induced by drought treatment, and 704 were downregulated after one week of drought treatment. However, only 261 unigenes were functionally annotated with GO terms (Figure 3A-C), and only 207 unigenes were identified in the KEGG pathway database (Figure 3D). Interestingly, approximately $12.1 \%$ of the unigenes with known function fell into the categories related to fatty acid metabolism, starch and sucrose metabolism, and nitrogen metabolism (Figure 3D), suggesting that these pathways and processes may participate in the drought response.

Previous studies have shown that transcription factors have a major effect on the network of drought-response genes. In this study, a total of 35 unigenes encoding known or putative transcription factors were found, including the WRKY, MYB, and ethylene-responsive transcription factors (Additional file 4).

\section{Expression of selected genes differentially regulated between the two DGE libraries}

To confirm the gene expression data, 18 unigenes whose expression was upregulated in drought plants were randomly chosen from the two libraries for qRTPCR analysis. Among them, 11 unigenes were significantly upregulated in drought-treated plants, while the expression levels of the remaining 7 unigenes were not significantly different between the two libraries. As shown in Figure 4, the unigene expression trends were similar in both DGE sequencing and qRT-PCR data. After examining the gene annotation information available for these unigenes, it was found that information on gene function was available for three unigenes; however, no matches in any database were obtained for the remaining 8 unigenes. These results suggest that some drought-resistance pathway genes may work together to defend the plant from drought stress.

\section{Development and characterisation of EST-SSR markers}

To further evaluate the quality of the sequence data assembly and to develop new molecular markers, the 79,918 unigenes generated in this study were used to mine potential microsatellites that were defined as mono- to hexanucleotide motifs with a minimum of three repetitions. Using the MISA software (http://pgrc.ipk-gatersleben.de/ misa/misa.html), a total of 17,310 potential simple sequence repeats (SSRs) were identified in 13,840 unigenes. Of these 13,840 unigenes, 11,078 and 2,762 unigenes contained one or more than one SSR, respectively (Table 3). The number of potential EST-SSRs per unigene varied from 1 to 8 , with an average of 1.25.

Using the SSR-containing sequences, 113 SSR sites were randomly selected to design EST-SSR primers with the Primer Premier 3.0 software. Information about these EST-SSR primers is shown in Additional file 5. Among these 113 primer pairs, 96 were used successfully to PCRamplify genomic DNA (Figure 5), while the remaining seventeen pairs of primers failed to generate PCR products at several annealing temperatures. Among the PCR 


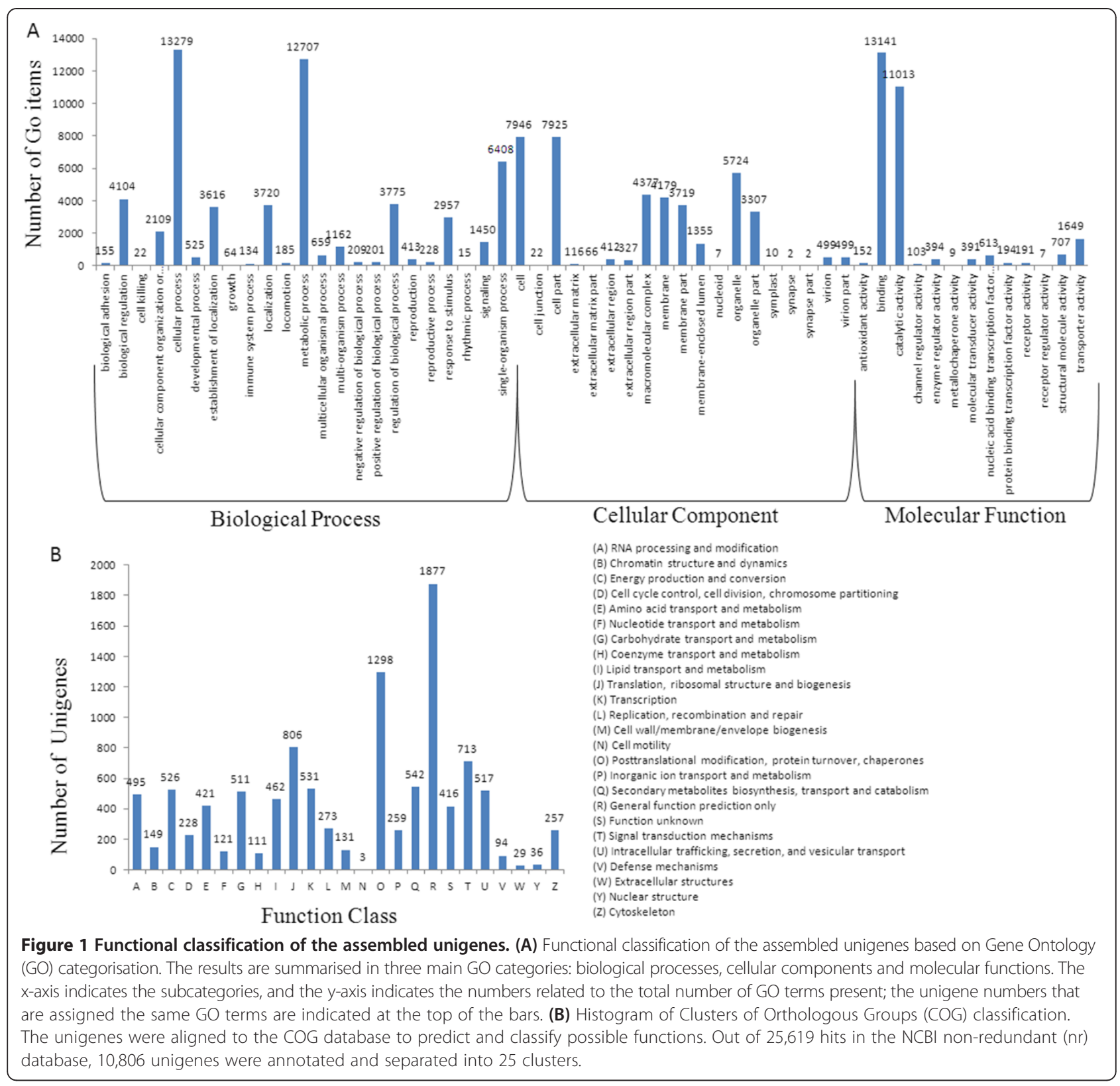

products of the 96 working primer pairs, 87 PCR products appeared to result from specific amplification; among these 87 PCR products, 78 PCR products were of the expected sizes, while the other six PCR products were larger than the expected sizes, suggesting that the amplified regions likely contained introns. Nine primer sets yielded PCR products that separated into more than one band, which may have resulted from either the primer design or the high heterozygosity of the $H$. ammodendron germplasm.

\section{Discussion}

In this study, a large number of $H$. ammodendron transcriptomic unigenes $(79,918)$ were sequenced using the
Illumina HiSeq 2000 platform (Table 1). The N50 length of the unigenes was $1,345 \mathrm{bp}$, and the average length was $728 \mathrm{bp}$; these results were comparable to those obtained in recently published transcriptomic analyses of other plant species, such as Reaumuria soongorica (N50 = 1,109 bp, average length $=677$ bp [25]) and Litchi $(\mathrm{N} 50=811$ $\mathrm{bp}$, average length $=601 \mathrm{bp}[26]$ ). To date, Trinity is one of most powerful software packages used for the de novo assembly of short reads. In this study, fewer than half of the unigenes $(29,989,37.52 \%)$ identified were successfully annotated using BLAST searches of the public nr, nt, SWISS-PROT, GO, COG and KEGG databases, given the absence of genomic information on $H$. ammodendron (Table 2). Notably, the percentage of unigenes that were 


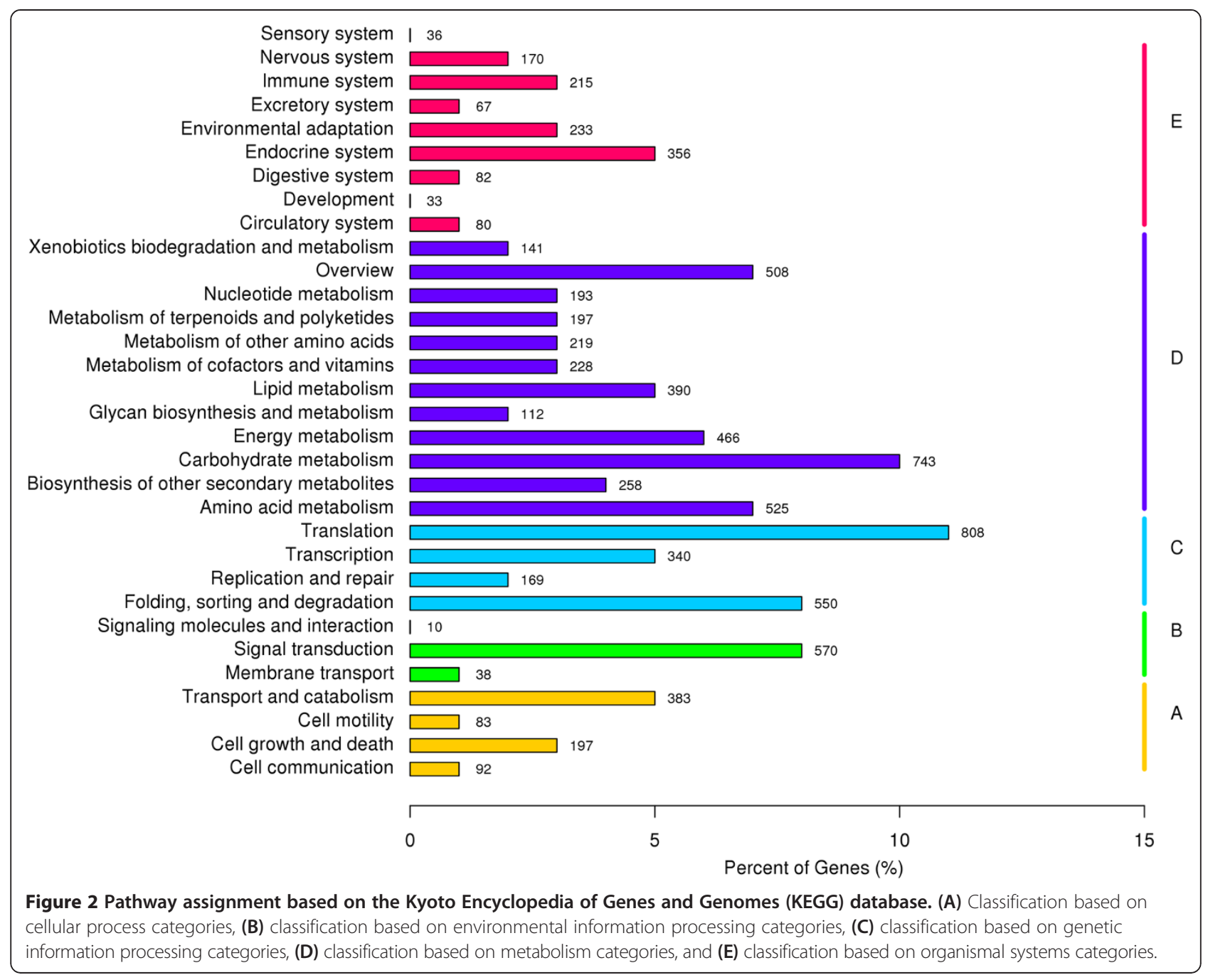

annotated is the lowest among previous studies conducted using the same sequencing strategy during the previous year (55 to $78.9 \%,[25,27-29])$. It is possible that a larger percentage could not be annotated in this study due to technical limitations, such as sequencing depth or read length [30], that are common to all studies that perform de novo transcriptome analyses. We found that the unannotated sequences were, on average, much shorter than the annotated unigenes (402 bp vs. $975 \mathrm{bp}$ ).

The C4 pathway has been acknowledged to be more adaptive than the $\mathrm{C} 3$ pathway in response to abiotic stresses, such as high temperature, radiation and drought [31].

H. ammodendron is a C4 plant, according to its physiological characteristics [31]. In this study, most of the genes encoding key enzymes involved in the $\mathrm{C} 4$ carbon fixation pathway were presented in the transcriptomic dataset from the annotation of the KEGG pathway. The gene expression data confirmed the $\mathrm{C} 4$ character of $\mathrm{H}$. ammodendron. The $17 \mathrm{C} 4$ pathway genes that were identified were all downregulated in drought-treated plants compared with the controls. This result is consistent with the previous finding that the efficiency of the photosystem decreases in conditions of water deficiency.

It has long been known that extensive changes in gene expression occur when plants are exposed to drought stress [1]. Generally, both upregulation and downregulation of gene expression occur under drought conditions. It has been reported that more genes are upregulated than are downregulated under drought stress in model plants [32]. In A. thaliana, 16,744 genes have been found to be drought responsive; it is interesting to note that after $2 \mathrm{~h}$ of treatment, 1,188 (7\%) were found to be upregulated and 217 (1.3\%) downregulated, while after $10 \mathrm{~h}$ of treatment, nearly the same percentage of genes (12.3\%) was upregulated as was downregulated [3]. In cotton, over $16 \%$ of the genome exhibited altered expression levels in response to drought stress. Among the genes whose expression levels were altered, 5,344 genes were induced by drought shock, and 4,630 were downregulated 
with 2 days of treatment [33]. In the present study, 1,060 differentially expressed genes were identified, but the proportion of the genome that is devoted to drought stress is unknown due to a lack of genome resources for this species. Approximately half of the genes examined were found to be drought-inducible. These results suggest that plants vary in their abilities to adapt to drought stress. Gene expression in desert plants may differ extensively from that in inland plants, and the differences in desert plants' gene expression patterns may confer an enhanced ability to respond to drought stimuli. Among the differentially expressed genes regulated by drought stress in $H$. ammodendron, over $50 \%$ had no homologues in the NCBI database. Some of these genes may represent novel drought-responsive transcripts that have not been reported in other plants.
EST-SSR markers are very important for research on a variety of topics, including the assessment of genetic diversity, the development of genetic maps, comparative genomics, marker assisted selection breeding, and others. To our knowledge, no previous study has reported the identification of ESR-SSRs in this desert tree. Transcriptome sequencing provided a large number of sequences that could be used to develop EST-SSR markers in the $H$. ammodendron tree. In total, 17,310 potential EST-SSRs were identified in 13,840 unigenes. In this study, in addition to the more common dinucleotide, trinucleotide and other nucleotide repeats that were included in the selection, mononucleotide repeat SSRs were also included, and the proportion of EST-SSRs that were mononucleotide repeats was larger than those of the other types of repeats. Trinucleotide repeats were the next-most abundant 


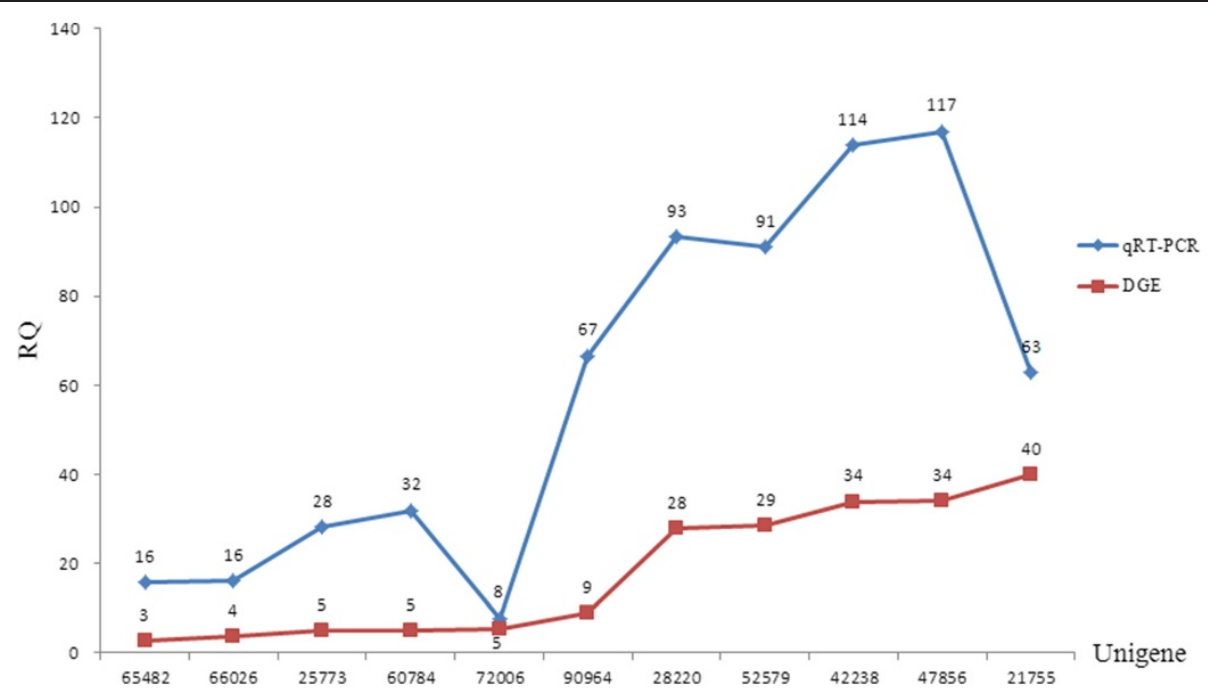

Figure 4 Unigene expression tendencies in both DGE sequencing data and qRT-PCR experimental results. The x-axis shows the different unigenes, and the $y$-axis represents the drought quantity relative to control levels. The numbers shown above the two graphs indicate the fold changes for each unigene for the drought treatment relative to control conditions.

type, followed by dinucleotide repeats, consistent with previous reports [34]. The most abundant dinucleotide and trinucleotide motifs were AG/TC and AAG/TTC, respectively. These results are consistent with previous results for dicots such as oak trees [35] and castor bean plants [36]. Of 113 primer pairs randomly selected for PCR validation, 96 (85\%) produced clear bands. The PCR success rate was similar to that observed in several previous studies, such as in a study of poplar trees [34], but higher than that reported in a study by Triwitayakorn et al. (75\%) [37]. Therefore, the 17,310 potential EST-SSRs identified in this study will provide a wealth of resources for developing EST-SSRs in the desert tree.

Table 3 Summary of the EST-SSRs that were identified in the transcriptome

\begin{tabular}{lr}
\hline Search item & Number \\
\hline Total number of examined unigenes & 79,918 \\
Total size of examined sequences (bp) & $58,206,305$ \\
Total number of identified EST-SSRs & 17,310 \\
Number of EST-SSRs containing sequences & 13,840 \\
Number of sequences containing more than one EST-SSR & 2101 \\
Mononucleotide & 10,630 \\
Dinucleotide & 2316 \\
Trinucleotide & 3969 \\
Tetranucleotide & 351 \\
Pentanucleotide & 23 \\
Hexanucleotide & 21 \\
\hline
\end{tabular}

\section{Conclusions}

In this study, we used high-throughput sequencing data to characterise the transcriptome of $H$. ammodendron, a species for which few genomic data are available. DGE sequences were mapped to the assembled transcriptome for further gene expression analysis. A large number of candidate genes involved in drought stress were identified. Furthermore, a set of EST-SSRs were identified that have specific PCR products. This data represents a fully characterised transcriptome and provides a valuable resource for genetic and genomic studies in desert plants.

\section{Methods}

\section{Sample collection and preparation}

$H$. ammodendron seeds were provided by the Gansu Desert Control Institute. The seeds were sowed on damp filter papers and incubated at $4^{\circ} \mathrm{C}$ for 4 days before being placed at $23^{\circ} \mathrm{C}$ under long-day ( $16 \mathrm{~h}$ light $/ 8 \mathrm{~h}$ dark) conditions with a photosynthetic photon flux density of $150 \mu \mathrm{mol} \mathrm{m} \mathrm{m}^{-2} \mathrm{~s}^{-1}$. The seedlings were grown in four pots (20 seedlings/pot) representing two replicates of two treatments. After the seedlings grew for one month, one set of seedlings were treated with a one-week (7d) stress, and the second set of seedlings was used as a control and received no treatment. The control pots were irrigated from the bottom every day, while drought-stressed pots were not irrigated and were monitored for wilting symptoms. We checked the drought phenotype when treated without watering, and found that the seedlings were wilting for one week not watering. So we chose this condition for finding the genes response to drought 


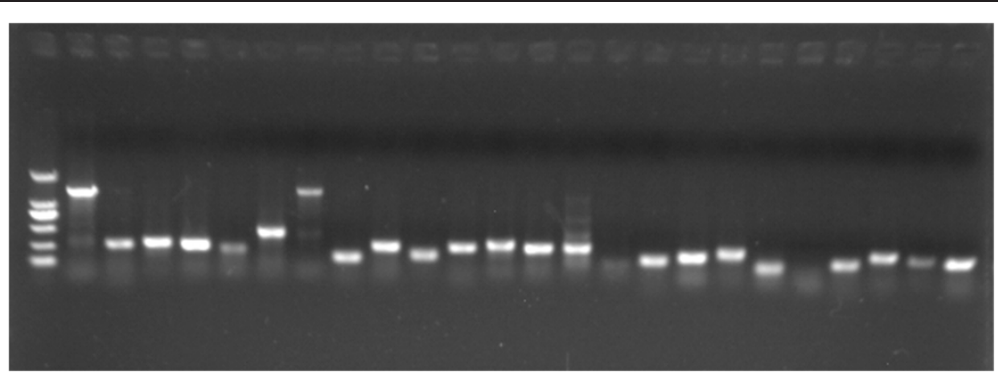

Figure 5 Photograph of PCR amplification results for the EST-SSR markers in H. ammodendron. The first line is the DNA ladder. The subsequent lines are the PCR products generated using different primers.

stress. Several tissues, including leaves, stems and roots, were then harvested from the drought and control samples for subsequent RNA isolation.

\section{RNA isolation and transcriptome sequencing}

Total RNA was extracted from the two replicates of the drought and control plants with TRIzol Reagent (Invitrogen, 15596-026) according to the manufacturer's instructions. The four RNA samples that were of sufficient quality were used to construct the transcriptome sequence library. The total four RNA from each sample was then pooled to one, using equivalent quantities of each sample. The transcriptome sequencing library was generated using NEBNext Ultra RNA Library Prep Kits for Illumina(NEB, USA) following manufacturer's instructions. Following the instructions provided by Illumina, mRNA was purified from the pooled, total RNA using polyT oligo-attached magnetic beads (Novogene, China). Fragmentation buffer was added to disrupt the mRNA into short fragments. Reverse transcriptase and random primers were used to synthesise the first strand cDNA from the cleaved mRNA fragments. The second strand cDNA was synthesised using buffer, dNTPs, RNase $\mathrm{H}$, and DNA polymerase I. The double strand cDNA was purified using QIAquick PCR extraction kits (QIAGEN, Hilden, Germany) and washed with EB buffer for end repair and single nucleotide A (adenine) addition. Finally, sequencing adaptors were ligated onto the fragments. The required fragments were purified by AMPure XP beads and enriched by PCR to construct a library for transcriptome sequencing.

\section{Data filtering and de novo sequence assembly}

The transcriptome library was sequenced using the Illumina HiSeq 2000 system. The sequencing-received raw image data were transformed by base calling into raw sequence data, which were termed raw reads. The raw data were then filtered by data-processing steps to generate clean data via a process that included the removal of adapter sequences, reads in which unknown bases are greater than 10\%, and low-quality sequences (in which the percentage of low-quality bases of quality value $\leq 5$ is greater than $50 \%$ in a read). After the clean data was generated, transcriptome assembly was accomplished using Trinity software [38] with min_kmer_cov set to 2 by default and all other parameters set to default values. The raw data are available in the Gene Expression Omnibus (GEO; http://www.ncbi.nlm.nih.gov/projects/ geo/) under accession number GSE63970.

\section{Functional annotation of unigenes}

For functional annotation, the assembled unigenes that might putatively encode proteins were searched against the nr (http://www.ncbi.nlm.nih.gov/), SWISS-PROT (http://www.expasy.ch/sprot/), KEGG (http://www.genome. jp/kegg/) and COG (http://www.ncbi.nlm.nih.gov/cog/) databases using the BLASTX algorithm. A typical cut-off value of E-value $<1$ e-5 was used. With $\mathrm{Nr}$ annotations, the Blast2GO program [39] was used to assign GO annotations to the unigenes according to component function, biological process and cellular component ontologies. After getting GO annotations for all unigenes, WEGO software [40] was used to assign GO functional classifications to all the unigenes and to understand the distribution of gene functions for the species on the macro level.

\section{DGE library preparation, sequencing and mapping analysis}

Total RNA from different tissues, including leaves, stems, and roots, were extracted from drought-treated and control plants for two replicates. Each DGE library included pooled RNA from 4 plants. A total of $3 \mu \mathrm{g}$ RNA per sample was used as input material for the RNA sample preparations. The procedure for constructing the DGE sequencing libraries was the same as that for constructing the transcriptome sequencing libraries. After the raw data was generated and the data-processing steps were completed, the clean reads were then mapped to the assembly transcriptome reference sequences using RSEM software [41]. Mismatches of no more than 2 bases were allowed in the alignments. The read count for each gene was obtained from the mapping results. 
The DGE data are available in the Gene Expression Omnibus (GEO; http://www.ncbi.nlm.nih.gov/projects/ geo/) under accession number GSE63970.

\section{Identification of differentially expressed unigenes}

Gene expression levels were calculated based on the numbers of reads mapped to the reference sequence, using the FPKM [42] method. After calculating gene expression levels, the differentially expressed genes (DEGs) were screened by comparing gene expression levels. Implementing the method described by Anders [43], differential expression analysis of two conditions was performed using the DESeq R package (1.10.1). DESeq provides statistical routines for determining differential expression in digital gene expression data using a model based on the negative binomial distribution. The resulting $\mathrm{P}$ values were adjusted using Benjamini and Hochberg's approach for controlling the false discovery rate. In this study, unigenes with an adjusted $\mathrm{P}<0.1$ found by DESeq were considered differentially expressed.

\section{Quantitative real-time PCR validation}

To confirm the DGE results, quantitative real-time reverse transcription PCR (qRT-PCR) was performed. Eighteen unigenes were randomly chosen for qRT-PCR analysis in the two libraries. The primers employed in the qRT-PCR experiments are listed in Additional file 6. qRT-PCR was implemented using the SYBR premix Ex Taq kit (TaKaRa, Dalian, China) on an ABI 7500 Real-Time System (Applied Biosystems), with the first strand cDNA serving as the template. Eighteen transcripts were randomly chosen for qRT-PCR analysis. The assembled Actin unigene (comp15413_C0) was used as an internal control. The relative quantitative method $(\Delta \Delta \mathrm{CT})$ was used to calculate the fold change in the expression levels of target genes [44]. All reactions were performed in three technical replicates using one biological sample.

\section{Development and detection of EST-SSR markers}

The MISA software (http://pgrc.ipk-gatersleben.de/ misa/misa.html) was used to identify microsatellites in the unigenes. In this study, EST-SSRs were considered to contain motifs consisting of one to six nucleotides. Primers for each SSR were designed using Primer3 software (http://primer3.ut.ee). In total, 113 pairs of primers were designed (Additional file 5) and validated by PCR analysis. The DNA for PCR amplification was extracted from the control samples using the CTAB method [45]. PCR amplification was conducted as follows: PCR mixtures were held at $94^{\circ} \mathrm{C}$ for $4 \mathrm{~min}$, followed by $35-40$ cycles of $94^{\circ} \mathrm{C}$ for $30 \mathrm{~s}, 55-60^{\circ} \mathrm{C}$ for 30 $\mathrm{s}$ and $72^{\circ} \mathrm{C}$ for $30 \mathrm{~s}$. The final extension was performed at $72^{\circ} \mathrm{C}$ for $10 \mathrm{~min}$. The PCR products were analysed by electrophoresis on $1.0 \%$ agarose gels.

\section{Additional files}

Additional file 1: Length distributions of assembly transcripts and unigenes.

Additional file 2: Summary of the GO classifications of assembled unigenes.

Additional file 3: Summary of the KEGG classifications of assembled unigenes.

Additional file 4: Transcription factors identified among the DEGs.

Additional file 5: Primer information for EST-SSRs.

Additional file 6: Primer information for qRT-PCR analysis.

Competing interests

The authors declare that they have no competing interests.

\section{Authors' contributions}

This study was conceived by YL, JWZ and XWP. The preparation of plant material was conducted by SSW and XJT, library construction and gene-expression analyses were conducted by QZ and JPZ. XJT and YL contributed to the data analysis, the bioinformatics analysis and manuscript preparation. ZHD and XWP revised the manuscript. All authors read and approved the final manuscript.

\section{Acknowledgements}

This work was supported by grants from the National Special Program for Transgenic Research (Nos. 2014ZX08011-001).

Received: 11 August 2014 Accepted: 11 December 2014 Published: 15 December 2014

\section{References}

1. Shanker AK, Maheswari M, Yadav SK, Desai S, Bhanu D, Attal NB, Venkateswarlu B: Drought stress responses in crops. Funct Int Genom 2014, 14(1):11-22.

2. Golldack D, Li C, Mohan H, Probst N: Tolerance to drought and salt stress in plants: unraveling the signaling networks. Front Plant Sci 2014, 5:151

3. Matsui A, Ishida J, Morosawa T, Mochizuki Y, Kaminuma E, Endo TA, Okamoto M, Nambara E, Nakajima M, Kawashima M, Satou M, Kim JM, Kobayashi N, Toyoda T, Shinozaki K, Seki M: Arabidopsis transcriptome analysis under drought, cold, high-salinity and $\mathrm{ABA}$ treatment conditions using a tiling array. Plant Cell Physiol 2008, 49(8):1135-1149.

4. Jeong JS, Kim YS, Baek KH, Jung H, Ha SH, Do Choi Y, Kim M, Reuzeau C, Kim JK: Root-specific expression of OsNAC10 improves drought tolerance and grain yield in rice under field drought conditions. Plant Physiol 2010, 153(1):185-197.

5. Ren X, Chen Z, Liu Y, Zhang H, Zhang M, Liu Q, Hong X, Zhu JK, Gong Z: ABO3, a WRKY transcription factor, mediates plant responses to abscisic acid and drought tolerance in Arabidopsis. Plant J 2010, 63(3): 417-429.

6. Cominelli E, Sala T, Calvi D, Gusmaroli G, Tonelli C: Over-expression of the Arabidopsis AtMYB41 gene alters cell expansion and leaf surface permeability. Plant J 2008, 53(1):53-64.

7. Chen CD, Zhang LY, Hu WK: The basic characteristics of plant communities, flora and their distribution in the sandy district of Gurbantungut. Acta Phytoecol Geobot Sin 1983, 7:89-99.

8. Tobe $K$, Li XM, Omasa K: Effects of sodium chloride on seed germination and growth of two Chinese desert shrubs, Haloxylon ammodendron and H-persicum (Chenopodiaceae). Aust J Bot 2000, 48(4):455-460.

9. Ma HB, Bao GX, Ma WD, Rong ZJ, Wang XY, Li B: The resource,protection and utilization of Haloxylon ammodendron deserted grassland in Inner Mongolia. Pratacult Sci 2000, 17:1-5.

10. Gao S, Su P, Yan Q, Ding S: Canopy and leaf gas exchange of Haloxylon ammodendron under different soil moisture regimes. Sci China Life Sci 2010, 53(6):718-728. 
11. Xie TT, Zhang XM, Liang SM, Shan LS, Yang XL, Hua YH: Effects of different irrigations on the water physiological characteristics of Haloxylon ammodendron in Taklimakan Desert hinterland. Ying Yong Sheng Tai Xue Bao 2008, 19(4):711-716.

12. Yang YF, Zhou HF, Xu LG: Dynamic variations of soil moisture in Haloxylon ammodendron root zone in Gurbantunggut Desert. Ying Yong Sheng Tai Xue Bao 2011, 22(7):1711-1716.

13. Shi L, Gan XY, Xia XL, Chen YC, Li M, Song YX: Cloning of choline monooxygenase gene from Haloxylon ammodendron and construction of its plant expression vector. Acta Bot Boreali Occidentalia Sin 2010, 30 (8):1514-1519.

14. Zhou $X Y$, Shi L, Gan $X Y$, Chen $Y C$, Song $Y X$ : Cloning and sequence analysis of ADP-ribosylation factors genes from Haloxylon ammodendron. Acta Agricult Boreali Occidentalis Sin 2012, 21(7):67-71.

15. Zhou XY, Gan XY, Shi L, Chen YC, Li M, Song YX: Cloning and sequence analysis of EF-hand CaBP gene from Haloxylon ammodendron. Acta Bot Boreali Occidentalia Sin 2012, 21(6):47-52.

16. Hegedus Z, Zakrzewska A, Agoston VC, Ordas A, Racz P, Mink M, Spaink HP Meijer AH: Deep sequencing of the zebrafish transcriptome response to mycobacterium infection. Mol Immunol 2009, 46(15):2918-2930.

17. Tian DQ, Pan XY, Yu YM, Wang WY, Zhang F, Ge YY, Shen XL, Shen FQ, Liu $\mathrm{XJ}$ : De novo characterization of the Anthurium transcriptome and analysis of its digital gene expression under cold stress. BMC Genomics 2013, 14:827.

18. Yates SA, Swain MT, Hegarty MJ, Chernukin I, Lowe M, Allison GG, Ruttink T, Abberton MT, Jenkins G, Skot L: De novo assembly of red clover transcriptome based on RNA-Seq data provides insight into drought response, gene discovery and marker identification. BMC Genomics 2014, 15:453.

19. Yu SC, Zhang FL, Yu YJ, Zhang DS, Zhao XY, Wang WH: Transcriptome profiling of dehydration stress in the Chinese Cabbage (Brassica rapa $\mathrm{L}$. ssp pekinensis) by Tag sequencing. Plant Mol Biol Report 2012, 30(1):17-28

20. Naegele R, Tomlinson AJ, Hausbeck MK: Evaluation of a diverse, worldwide collection of wild, cultivated and landrace peppers (Capsicum annuum) for resistance to Phytophthora fruit rot, genetic diversity and population structure. Phytopathology 2014, 105(1):110-118.

21. Ahmad Z, Mumtaz AS, Ghafoor A, Ali A, Nisar M: Marker Assisted Selection (MAS) for chickpea Fusarium oxysporum wilt resistant genotypes using PCR based molecular markers. Mol Biol Rep 2014, 41(10):6755-6762.

22. Talukder ZI, Gong L, Hulke BS, Pegadaraju V, Song Q, Schultz Q, Qi L. A high-density SNP map of sunflower derived from RAD-sequencing facilitating fine-mapping of the rust resistance gene R12. PLOS One 2014, 9(7):e98628.

23. Li DJ, Deng Z, Qin B, Liu XH, Men ZH: De novo assembly and characterization of bark transcriptome using Illumina sequencing and development of EST-SSR markers in rubber tree (Hevea brasiliensis Muell. Arg.). BMC Genomics 2012, 13:192.

24. Zhao Y, Williams R, Prakash C, He G: Identification and characterization of gene-based SSR markers in date palm (Phoenix dactylifera L.). BMC Plant Biol 2012, 12(1):237.

25. Shi Y, Yan X, Zhao P, Yin H, Zhao X, Xiao H, Li X, Chen G, Ma XF: Transcriptomic analysis of a tertiary relict plant, extreme xerophyte Reaumuria soongorica to identify genes related to drought adaptation. PLoS One 2013, 8(5):e63993.

26. Li C, Wang Y, Huang $X$, Li J, Wang H, Li J: De novo assembly and characterization of fruit transcriptome in Litchi chinensis Sonn and analysis of differentially regulated genes in fruit in response to shading. BMC Genomics 2013, 14:552.

27. Liu M, Qiao G, Jiang J, Yang H, Xie L, Xie J, Zhuo R: Transcriptome sequencing and de novo analysis for Ma bamboo (Dendrocalamus latiflorus Munro) using the Illumina platform. PLoS One 2012, 7(10):e46766.

28. Lulin $H$, Xiao Y, Pei $S$, Wen $T$, Shangqin $H$ : The first Illumina-based de novo transcriptome sequencing and analysis of safflower flowers. PLOS One 2012, 7(6):e38653.

29. Xu DL, Long H, Liang JJ, Zhang J, Chen $X$, Li JL, Pan ZF, Deng GB, Yu MQ: De novo assembly and characterization of the root transcriptome of Aegilops variabilis during an interaction with the cereal cyst nematode. BMC Genomics 2012, 13:133.
30. Novaes E, Drost DR, Farmerie WG, Pappas GJ Jr, Grattapaglia D, Sederoff RR, Kirst M: High-throughput gene and SNP discovery in Eucalyptus grandis, an uncharacterized genome. BMC Genomics 2008, 9:312.

31. Zhao AF, Zhang LX, Du MW, Chen HS: Characteristic in photosynthesis, transpiration and water use effiency of Haloxylon ammodendron and Calligonum mongolicum of desert species. Acta Bot Boreali Occidentalis Sin 2003, 23(1):11-17.

32. Kreps JA, Wu Y, Chang HS, Zhu T, Wang X, Harper JF: Transcriptome changes for Arabidopsis in response to salt, osmotic, and cold stress. Plant Physiol 2002, 130(4):2129-2141.

33. Chen Y, Liu Z-H, Feng L, Zheng Y, Li D-D, Li X-B: Genome-wide functional analysis of cotton (Gossypium hirsutum) in response to drought. PLOS ONE 2013, 8(11):e80879.

34. Du FK, Xu F, Qu H, Feng S, Tang J, Wu R: Exploiting the transcriptome of Euphrates Poplar, Populus euphratica (Salicaceae) to develop and characterize new EST-SSR markers and construct an EST-SSR Database. PLOS ONE 2013, 8(4):e61337.

35. Durand J, Bodenes C, Chancerel E, Frigerio JM, Vendramin G, Sebastiani F, Buonamici A, Gailing O, Koelewijn HP, Villani F, Mattioni C, Cherubini M, Goicoechea PG, Herran A, Ikaran Z, Cabane C, Ueno S, Alberto F, Dumoulin PY, Guichoux E, de Daruvar A, Kremer A, Plomion C: A fast and cost-effective approach to develop and map EST-SSR markers: oak as a case study. BMC Genomics 2010, 11(1):570.

36. Qiu L, Yang C, Tian B, Yang J-B, Liu A: Exploiting EST databases for the development and characterization of EST-SSR markers in castor bean (Ricinus communis L.). BMC Plant Biol 2010, 10(1):278.

37. Triwitayakorn K, Chatkulkawin P, Kanjanawattanawong S, Sraphet S, Yoocha T, Sangsrakru D, Chanprasert J, Ngamphiw C, Jomchai N, Therawattanasuk K, Tangphatsornruang S: Transcriptome sequencing of Hevea brasiliensis for development of microsatellite markers and construction of a genetic linkage map. DNA Res 2011, 18(6):471-482.

38. Grabherr MG, Haas BJ, Yassour M, Levin JZ, Thompson DA, Amit I, Adiconis X, Fan L, Raychowdhury R, Zeng QD, Chen Z, Mauceli E, Hacohen N, Gnirke A, Rhind N, di Palma F, Birren BW, Nusbaum C, Lindblad-Toh K, Friedman N, Regev A: Full-length transcriptome assembly from RNA-Seq data without a reference genome. Nat Biotechnol 2011, 29(7):644-U130.

39. Conesa A, Gotz S, Garcia-Gomez JM, Terol J, Talon M, Robles M: Blast2GO: a universal tool for annotation, visualization and analysis in functional genomics research. Bioinformatics 2005, 21(18):3674-3676.

40. Ye J, Fang L, Zheng H, Zhang Y, Chen J, Zhang Z, Wang J, Li S, Li R, Bolund L, Wang J: WEGO: a web tool for plotting GO annotations. Nucleic Acids Res 2006, 34(Web Server issue):W293-W297.

41. Li B, Dewey CN: RSEM: accurate transcript quantification from RNA-Seq data with or without a reference genome. BMC Bioinf 2011, 12:323.

42. Harwood S: Vector NTI. Biotechnol Softw I J 1996, 13(5):22-30.

43. Anders S, Huber W: Differential expression analysis for sequence count data. Genome Biol 2010, 11(10):R106.

44. Quail MA, Kozarewa I, Smith F, Scally A, Stephens PJ, Durbin R, Swerdlow H, Turner DJ: A large genome center's improvements to the Illumina sequencing system. Nat Methods 2008, 5(12):1005-1010.

45. Del Sal G, Manfioletti G, Schneider C: The CTAB-DNA precipitation method: a common mini-scale preparation of template DNA from phagemids, phages or plasmids suitable for sequencing. BioTech 1989, 7(5):514-520.

doi:10.1186/1471-2164-15-1111

Cite this article as: Long et al:: De novo assembly of the desert tree Haloxylon ammodendron (C. A. Mey.) based on RNA-Seq data provides insight into drought response, gene discovery and marker identification. BMC Genomics 2014 15:1111. 